\title{
A case of mandible Paget's disease of the bone treated with intravenous neridronate
}

\author{
A. Fassio, L. Idolazzi, M. Rossini, O. Viapiana, D. Gatti \\ Unit of Rheumatology, University of Verona, Italy
}

\section{SUMMARY}

Paget's disease of bone (PDB) is a focal disorder of osteoclasts, leading to chaotic bone remodelling, and it is characterized by the presence of focal areas of excessive bone formation alongside with areas of focal bone resorption. The typical radiographic feature is the cotton wool appearance. To date, bisphosphonates are the mainstay of the treatment. We hereby report the case of a young woman presenting with mandible PDB, with a relevant diagnostic delay and mistakenly treated for five years with chronic oral corticosteroids. After our evaluation, the patient received treatment with intravenous neridronate (an amino-bisphosphonate licensed in Italy for the treatment of this disease), with achievement of clinical remission.

Key words: Paget's disease of the bone; neridronate; mandible.

\section{INTRODUCTION}

aget's disease of bone (PDB) is a focal disorder of bone remodelling that commonly occurs in older people and was first described by Sir James Paget under the term osteitis deformans in 1877 . It can be classified as monostotic (involving a single bone) or polyostotic (thus involving several bones of the body). The distinctive feature of PDB is the presence of focal bone remodelling with high bone turnover and with the typical cotton wool appearance radiographic sign (1). It is hypothesized that the primary abnormality might be an increased osteoclast activity. Osteoclasts prove to be enlarged in size and high in number (2). The newly formed bone is irregular, lacking the normal lamellar pattern and increased in volume, sclerotic and weaker than normal bone. The most common symptom is pain and the progression of PDB can lead to deformity and secondary complications. Because pagetic bone is enlarged and weaker than normal bone, it is associated with increased rate of osteoarthritis due to the altered joint dynamics, fragility fractures, bone deformity and spinal stenosis (3). When involving the jaw, facial disfigurement may be the conse- quence of enlargement of the maxilla and/ or mandible (4).

Although very rare, the development of osteosarcoma (especially in the elderly), is a worrisome complication (3).

$\mathrm{X}$-rays show the typical features of the disease, including focal lytic areas with a coarse trabecular pattern and increased cortical thickness. The bone itself may deform depending on the severity of the disease and type of bone involvement. Vertebrae usually appear enlarged in size with a typical squared appearance, while long bones may deform or also, in some cases, become bowed (5). Bone scintigraphy is useful to determine the disease extent and to differentiate the monostotic from the polyostotic forms (5). Bone turnover markers (BTM) also play an important role in diagnosis and in follow-up after appropriate treatment. BTMs are divided into markers of bone formation and of bone resorption. The former include: total alkaline phosphatase (ALP), bone alkaline phosphatase and the intact $\mathrm{N}$-propeptide of type I collagen. The latter include: serum collagen type I cross-linked C-telopeptide (CTX) and urine N-terminal telopeptide. Usually, due to its low cost and universal availability, total ALP is the most used marker. In patients with abnormal 
liver or biliary tract and in those who show normal ALP value, other markers may be considered (6).

Aminobisphosphonates are the mainstay of pharmacological treatment of PDB. Oral alendronate and risedronate have been used, but recent studies show better results with intravenous zoledronate (7-9). Neridonate has also proved to be effective and safe in the treatment of PDB (10) and is licensed in Italy for treatment of the disease.

\section{CASE REPORT}

We report the case of a 22-year-old woman who had been complaining of bilateral mandible pain since she was 10 years old. The clinical records started in 2010, when the patient had a referral for jaw pain in a suburban hospital. At that time, the clinical examination revealed a visible broadening and widening of the lower face. The patient underwent a bone scintigraphy showing a diffuse increased radionuclide uptake at the jaw (Figure 1). She also underwent a bone biopsy of the same tissue. The histologic report excluded any neoplastic process and documented the presence of diffuse foci of osteitis alongside diffuse reactive foci of bone repair with a final generic diagnosis of osteitis. Without a definite diagnosis, systemic and chronic corticosteroid therapy (from moderate to high dosages) was started.

In 2015, she attended our bone disease outpatient clinic for the first time. The patient was then still undergoing chronic corticosteroid therapy and the diagnosis was unclear.

The clinical examination still revealed broadening and widening of the mandible, but those findings were mostly hidden by the cushingoid appearance of the face due to the longstanding corticosteroid treatment. No local anomaly of the skin was observed.

We thus asked the pathology department for the microscope slides and had them re-examined by our expert bone disease pathologist, who excluded any neoplastic process and confirmed the presence of diffuse foci of osteitis alongside diffuse reac-

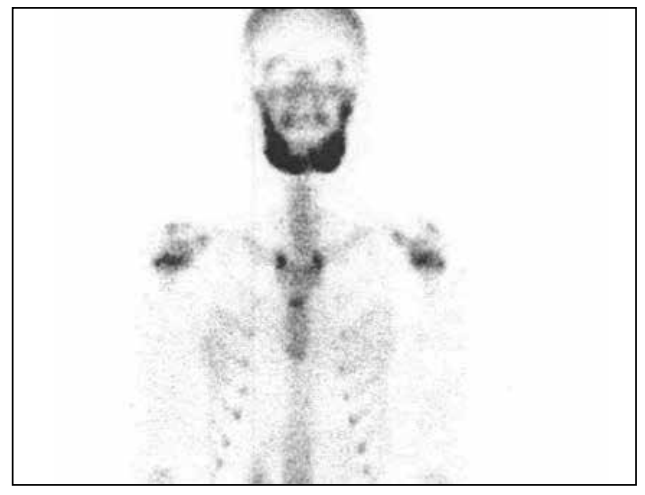

Figure 1 - Bone scan (99mTc-HMDP) anterior view, showing diffuse and severe increase of the radionuclide uptake of the tracer at the mandible bone.

tive foci of bone repair. Specifically, the pathologist described the presence of diffuse foci of bone remodelling together with an increased number of enlarged multinucleated osteoclasts. The presence of relevant fibrosis on the medullary spaces of the trabecular bone was also reported.

Maxillofacial X-rays and then a skull computed tomography (CT) were taken, providing evidence of increased cortical thickness of the body of the mandible and diffuse mixed lytic and sclerotic involvement. In addition, a severe deformity with flattening of the mandible condyles was described by the radiologist (Figure 2). An autologous white blood cells (WBC) scintigraphy was done in order to exclude the suspicion of a chronic infection. The bone scan revealed a symmetrical increase of the radionuclide uptake of the radio labelled bisphosphonate of the jaw, while the WBC scintigraphy resulted completely negative. The blood exams were all within ranges; among BTM, ALP was $59 \mathrm{U} / \mathrm{L}$ (ref. range 45-110 U/L), while CTX was $0.634 \mathrm{ng} / \mathrm{mL}$ (ref. range $0.150-0.450 \mathrm{ng} / \mathrm{mL}$ ) thus resulting slightly elevated.

Based on the clinical and imaging features, diagnosis of PDB was made and the patient received treatment with $100 \mathrm{mg}$ of intravenous neridronate on two consecutive administrations. Lastly, the steroid treatment was slowly diminished and suspended. In the months after the treatment most of the mandible pain improved. Due to the op- 


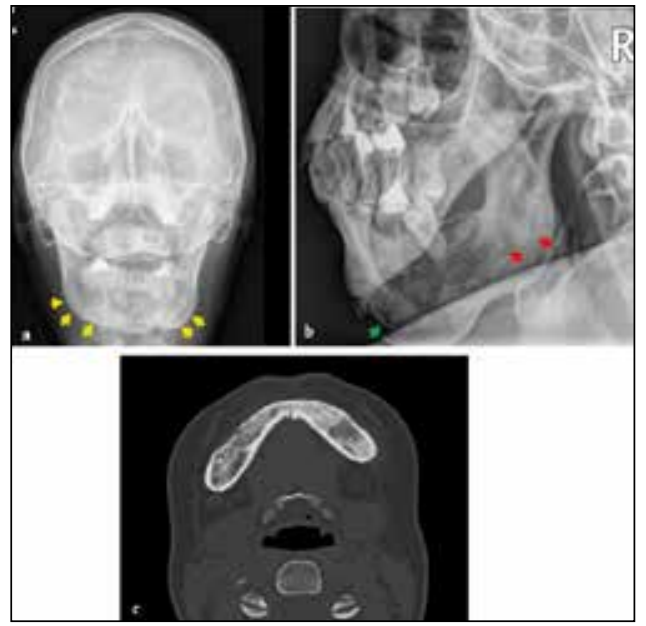

Figure 2 - A) and B) X-rays images and C) computed tomographic slice of the chin. These images show a widened mandible with irregular and thickened borders (yellow arrows) and with mixed lytic and sclerotic modifications of the bone texture (green and red arrows, respectively).

timal clinical response to the drug, no further invasive procedures were considered necessary. The patient is now doing well and is still in follow-up. One year later, the ALP and CTX values were both within the normal range (respectively $53 \mathrm{U} / \mathrm{L}$ and $0.37 \mathrm{ng} / \mathrm{mL}$ ).

\section{DISCUSSION}

Worldwide the prevalence of PDB varies substantially (3), from very low in Japan $(<0.01 \%)$ to high $(>1 \%)$ in Western Europe, Australia and New Zealand. The majority of patients with PDB have involvement of at least one bone out of the pelvis, sacrum, lumbar spine or proximal femur. Maxillofacial involvement has already been described $(4,11)$, but to our knowledge this is the first time in which successful treatment with neridronate for a maxillofacial localization has been described.

The diagnosis of PDB is usually made on clinical and imaging evidence with the support of elevated BTM. In this case they were within limits (ALP) or slightly elevated (CTX), possibly because of the limited bone extension of the disease. This is a reasonable hypothesis, even though cases with mandible involvement and elevated BTM have been described (11). Another factor, which could help to explain the nearly normal values of the BTM, might also be the previous longstanding treatment with corticosteroids.

The importance of BTM, especially in those cases in which they are elevated since the moment of the diagnosis, consists in the monitoring of the efficacy of treatment and in the early individuation of relapses. In some rare cases, bone biopsy might have a role. In this particular case, it was probably performed to exclude the possibility of a chronic osteomyelitis. The typical histology of PDB lesions shows increased osteoclasts, which are often enlarged and multinucleated, and disorganised woven bone $(2,3)$. In our case, the findings of the biopsy were consistent with the these features.

However, experienced radiologists and physicians generally have no difficulty in distinguishing the lesions of Paget's disease from other skeletal disorders and only very seldom is it necessary to obtain a bone biopsy to help make the definitive diagnosis (6).

PDB is a disease characterized by a focal dysregulation of the normal bone turnover, thus bisphosponates are the mainstay of the treatment. The distinctive feature of PDB is the large osteoclasts actively resorbing the bone. These cells seem to respond adequately to bisphosphonate therapy.

In this case, it is not possible to exclude ultimately the eventuality of a monostotic fibrous dysplasia (FD), as a differential diagnosis. However, we found a number of reasons to support the diagnosis of PDB. The radiographic appearance of craniofacial FD can be very similar to PDB. For instance, the cotton wool appearance has also been described in FB (12). CT scan is currently considered to be the preferred technique in the examination of the features of lesions suspected for FD, identified by typical observations such as ground glass appearance, sometimes along with an orange peel or fingerprint texture (12).

Furthermore, in our patient, the biopsy did not report features typical of FD such as, 
for example, a fibrous stroma with a highly cellularized fibroblastic representation. On the contrary, the presence of giant and multinuclear osteoclasts (described, in our case, as diffuse by our pathologist) leads more towards the diagnosis of PDB.

Only few randomized clinical trials have compared the safety and efficacy of different bisphosphonates in PDB treatment. Namely, a comparison between oral alendronate and intravenous pamidronate proved that bisphosphonates had similar efficacy in achieving biochemical remission in previously untreated patients, but in those who had formerly been treated with pamidronate, alendronate was more effective (13). In two other studies, a single intravenous dose of zoledronate proved more effective than a 2-month course of oral administration of risedronate in controlling bone turnover in the short (9) and long (7) term, therefore becoming the bisphosphonate of choice in the treatment of PDB (14). Neridronate is licensed in Italy for the treatment of Paget's disease, with a dose of $100 \mathrm{mg}$ given intravenously for 2 consecutive days $(10,15)$. The highest dose was the most effective and was associated with a $65 \%$ rate of full remission and a biochemical response (decrease of $75 \%$ ) in $95 \%$ of the patients (10). In a randomized study comparing neridronate and zoledronate, a single neridronate and zoledronate infusion showed a similar efficacy in achieving biochemical remission in up to $90 \%$ of patients who were non-responders to pamidronate (16). Even though the international guidelines do not currently take into consideration neridronate for the treatment of PDB (6), those results, in our opinion, support the usage of neridronate as being not inferior to zoledronate or the other recommended bisphosphonates.

Overall, this case could be relevant for a number of reasons. Firstly, X-rays remain to date a test of paramount importance; performing a radiographic exam in the first instance could potentially have avoided five years of erroneous and potentially harmful corticosteroid treatment by greatly facilitating the diagnosis. Secondly, even if the presentation of a mandible PDB is quite rare, this possibility should by no means be excluded. Thirdly, a correct patient history, physical examination and laboratory and imaging tests in PDB is almost always sufficient for making the correct diagnosis.

Acknowledgements: The authors would like to thank Prof. Silvano Adami, who bequeathed to us his love for research and truth.

Conflict of interests: The authors declare no conflict of interests.

\section{BIBLIOGRAFIA}

1. Bhargava P, Maki JH. Images in clinical medicine. "Cotton wool" appearance of Paget's disease. N Engl J Med. 2010; 363: e9.

2. Seitz S, Priemel M, Zustin J, et al. Paget's disease of bone: histologic analysis of 754 patients. J Bone Miner Res Off J Am Soc Bone Miner Res. 2009; 24: 62-9.

3. Bolland MJ, Cundy T. Paget's disease of bone: clinical review and update. J Clin Pathol. 2013; 66: 924-7.

4. Smith NH. Monostotic Paget's disease of the mandible presenting with progressive resorption of the teeth. Oral Surg Oral Med Oral Pathol. 1978; 46: 246-53.

5. Smith SE, Murphey MD, Motamedi K, et al. From the archives of the AFIP. Radiologic spectrum of Paget disease of bone and its complications with pathologic correlation. Radiogr Rev Publ Radiol Soc N Am Inc. 2002; 22: 1191-216.

6. Singer FR, Bone HG, Hosking DJ, et al. Paget's disease of bone: an endocrine society clinical practice guideline. J Clin Endocrinol Metab. 2014; 99: 4408-22.

7. Hosking D, Lyles K, Brown JP, et al. Longterm control of bone turnover in Paget's disease with zoledronic acid and risedronate. $\mathbf{J}$ Bone Miner Res Off J Am Soc Bone Miner Res. 2007; 22: 142-8.

8. Reid IR, Davidson JS, Wattie D, et al. Comparative responses of bone turnover markers to bisphosphonate therapy in Paget's disease of bone. Bone. 2004; 35: 224-30.

9. Reid IR, Miller P, Lyles K, et al. Comparison of a single infusion of zoledronic acid with risedronate for Paget's disease. N Engl J Med. 2005; 353: 898-908.

10. Adami S, Bevilacqua M, Broggini M, et al. Short-term intravenous therapy with neridronate in Paget's disease. Clin Exp Rheumatol. 2002; 20: 55-8.

11. Wang W-C, Cheng Y-SL, Chen C-H, et al. Paget's disease of bone in a Chinese patient: 
a case report and review of the literature. Oral Surg Oral Med Oral Pathol Oral Radiol Endod. 2005; 99: 727-33.

12. Mainville GN, Turgeon DP, Kauzman A. Diagnosis and management of benign fibroosseous lesions of the jaws: a current review for the dental clinician. Oral Dis. 2016 [Epub ahead of print].

13. Walsh JP, Ward LC, Stewart GO, et al. A randomized clinical trial comparing oral alendronate and intravenous pamidronate for the treatment of Paget's disease of bone. Bone. 2004; 34: 747-54.
14. Reid IR. Pharmacotherapy of Paget's disease of bone. Expert Opin Pharmacother. 2012; 13: 637-46.

15. Gatti D, Rossini M, Viapiana O, et al. Clinical development of neridronate: potential for new applications. Ther Clin Risk Manag. 2013; 9: 139-47.

16. Merlotti D, Gennari L, Martini G, et al. Comparison of different intravenous bisphosphonate regimens for Paget's disease of bone. J Bone Miner Res Off J Am Soc Bone Miner Res. 2007; 22: 1510-7. 